\title{
IMPACT OF ANNEALING ON PASSIVATION OF a-Si:H / c-Si HETEROSTRUCTURES
}

\author{
Stefaan De Wolf, ${ }^{1,2}$ Hiroyuki Fujiwara ${ }^{1}$ and Michio Kondo ${ }^{1}$ \\ ${ }^{1}$ Research Center for Photovoltaics, National Institute of Advanced Industrial Science and Technology, \\ 1-1-1 Umezono, Tsukuba, Ibaraki 305-8568, Japan \\ ${ }^{2}$ Now at University of Neuchâtel, Breguet 2, $\mathrm{CH}-2000$ Neuchâtel, Switzerland
}

\begin{abstract}
The a-Si:H / c-Si heterostructure, is an attractive solution to avoid the presence of highly recombinative metal contacts at the surfaces of $c$-Si based solar cells. To assure good interface passivation, insertion of a sandwiched thin device-grade intrinsic a-Si:H(i) film is recommended between substrate and doped a-Si:H layer. In this article we discuss our findings on the impact of lowtemperature post-deposition annealing on the passivation properties of such stacks: we have identified two fundamentally different recombination mechanisms that may critically affect heterostructure device performance. Firstly, for the intrinsic buffer layer, whereas abrupt a-Si:H I $c$-Si interfaces typically benefit from post deposition annealing, it is shown that this is not true when epitaxially grown Si material is present at the interface. Secondly, in case the buffer layer is covered with a doped a-Si:H overlayer, annealing may again be detrimental for the interface passivation. The latter is linked to the fact that the presence of such doped layer may lower the energy required for Fermi-level dependent $\mathrm{Si}-\mathrm{H}$ bond rupture in the underlying intrinsic buffer layer, resulting in enhanced interface recombination.
\end{abstract}

\section{INTRODUCTION}

Hydrogenated amorphous silicon (a-Si:H) films deposited on crystalline silicon (c-Si) surfaces have increasingly attracted attention over the past years. Initially it was discovered that such films may passivate electronically c-Si surfaces,[1] and that abrupt electronic heterojunctions can be obtained with such structures.[2] These two phenomena were exploited not much later for the development of a number of electronic devices, including solar cells. For the latter, soon it was recognized that the output parameters benefit substantially from inserting a few $\mathrm{nm}$ thin intrinsic a-Si:H(i) film between the doped amorphous emitter and $c$-Si substrate,[3] where direct deposition of doped a-Si:H films on $c$-Si surfaces was speculated to result in poor interface properties. Presently, for solar cells that feature such a heterostructures, impressive energy conversion efficiencies exceeding $22 \%$ have been reported for large area $\left(>100 \mathrm{~cm}^{2}\right)$ devices.[4] Despite these results, the physical understanding of the a-Si:H / c-Si interface passivation is not yet complete.

In this article, we summarize our findings on the correlation between the electronic passivation quality and the nature of the a-Si:H / c-Si interface. For this purpose, an annealing study of thin stacked doped a-Si:H films is presented where electronic passivation results are linked to material properties. These studies are useful for two reasons: Firstly, postdeposition annealing offers in a straightforward way a single parameter to vary both electronic and material properties of the samples under study. This may yield new physical insights. Secondly, such treatments may be beneficial for the structures under study. Hence, they are also relevant from device processing point of view.

\section{EXPERIMENTAL DETAILS}

For the experiments, $300-320 \mu \mathrm{m}$ thick relatively low resistivity boron-doped $(\sim 3.0 \Omega . \mathrm{cm})$ and phosphorusdoped $(\sim 0.7 \Omega . \mathrm{cm})$ high quality float zone (100) FZ-Si wafers have been used. Both substrate surfaces were mirror polished to eliminate the influence of substrate surface roughness on the passivation properties and to allow for SE measurements. For surface cleaning, the samples were first immersed in a $\left(\mathrm{H}_{2} \mathrm{SO}_{4}: \mathrm{H}_{2} \mathrm{O}_{2}\right)$ (4:1) solution for 10 min to grow a chemical oxide, which was followed by a rinse in de-ionized water. The oxide was then stripped off in a dilute HF solution (5\%) for $30 \mathrm{sec}$. After this the samples were immediately transferred to the load lock of the deposition system. For a-Si:H film deposition, a clustered multi-chamber parallel plate directplasma enhanced chemical vapor deposition (PECVD) system, consisting of separate chambers for $i-$, and doped layer deposition was used. The electrode-distance and -diameter were respectively $20 \mathrm{~mm}$ and $230 \mathrm{~mm}$. After transfer of the samples to the relevant deposition chambers and mounting at the top electrodes, the wafer surfaces were exposed to a 200 SCCM (SCCM denotes cubic centimeter per minute at STP) $\mathrm{H}_{2}$ flow for 20 min at a pressure of 0.5 Torr for temperature stabilization. During film deposition, all chambers were operated at radio frequency $(\mathrm{rf})(13.56 \mathrm{MHz})$ power and at a pressure of 0.5 Torr. For soft film deposition, the used power was consistently the minimum required to maintain stable plasmas. The value for $T_{\text {depo }}$ was varied from $105{ }^{\circ} \mathrm{C}$ to $255^{\circ} \mathrm{C}$. For intrinsic film deposition $20 \mathrm{SCCM} \mathrm{SiH} 4$ was used, whereas for boron doped films we used 10 SCCM $\mathrm{SiH}_{4}$, mixed in $30 \mathrm{SCCM} \mathrm{B} \mathrm{H}_{6}\left(4660 \mathrm{ppm}\right.$ in $\mathrm{H}_{2}$ ). No additional $\mathrm{H}_{2}$ dilution was used during deposition. To evaluate the surface passivation quality, identical films (or stacked films) were deposited on both wafer surfaces. After deposition, the samples were consecutively annealed in a vacuum furnace (30 $\mathrm{min}$, with annealing temperatures, 


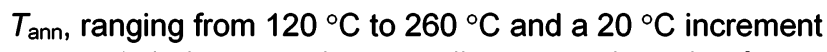
per step). In between the annealing steps, the value for $\tau_{\text {eff }}$ of the samples was measured with a Sinton Consulting WCT-100 quasi steady state photoconductance system,[5] operated in the generalized mode. Since high quality FZ-Si wafers have been used throughout the experiments, the contribution of the bulk to the total recombination expressed by $\tau_{\text {eff }}$ can be neglected. All reported values for $\tau_{\text {eff }}$ are evaluated at a constant minority carrier injection density, $\Delta n=\Delta p=1.0 \times 10^{15} \mathrm{~cm}^{-3}$. The thickness of the deposited films was ex-situ determined by measuring ellipsometry spectra $(\psi, \Delta)$ using a Woollam M-2000 rotating-compensator instrument. For bulk characterization of the films, thermal desorption spectroscopy (TDS) measurements were taken. For this an ESCO EMDWA1000S system operated at ultra high vacuum (< $1.0 \times 10^{-9}$ Torr) is used in which the samples are lampheated up to $1000^{\circ} \mathrm{C}$, with a linear temperature ramp of $20 \mathrm{~K} \cdot \mathrm{min}^{-1}$. During the annealing, a Balzers AG QMG 421 quadrupole mass spectrometer was used to determine the $\mathrm{H}_{2}$ effusion rate from the $\mathrm{a}-\mathrm{Si}: \mathrm{H}$ films. Throughout this article the following shorthand notations are used: $i, p^{+}$and $i / p^{+}$for respectively the $c-S i$ / a-Si:H(i), c-Si / a-Si:H $\left(p^{+}\right)$ and $c-\mathrm{Si} / \mathrm{a}-\mathrm{Si}: \mathrm{H}(I) / \mathrm{a}-\mathrm{Si}: \mathrm{H}\left(p^{+}\right)$structures.

\section{RESULTS}

Figure 1 shows how the surface passivation quality of a

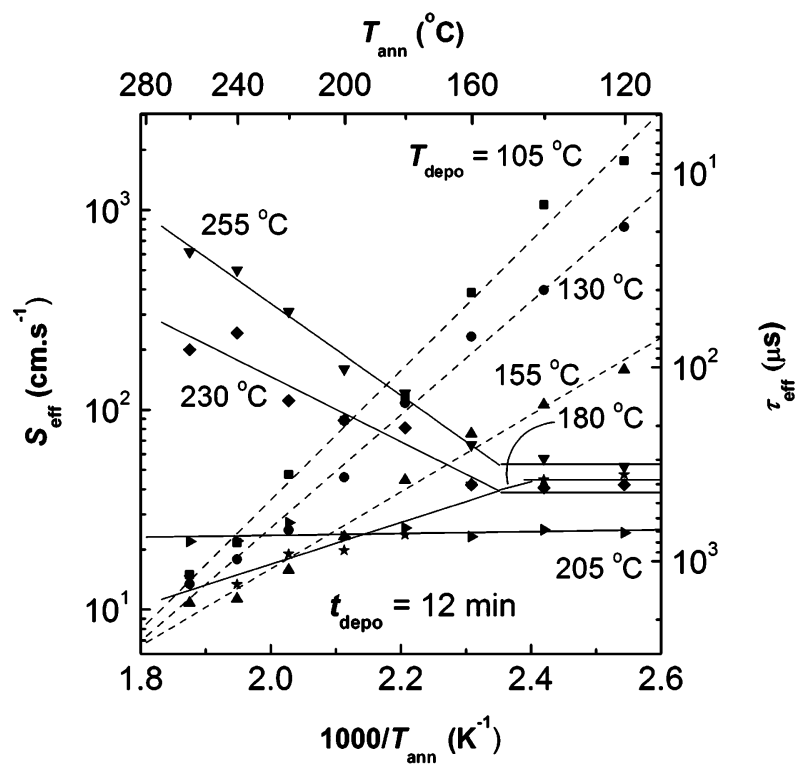

FIG. 1 Influence of $T_{\text {ann }}$ on the surface passivation quality for PECVD a-Si:H(i) films deposited on mirror polished (100) FZ-Si(p) surfaces at different temperatures, $T_{\text {depo }}$. All deposition times were $12 \mathrm{~min}$, whereas annealing times were $30 \mathrm{~min}$. Starting from the $T_{\text {ann }}$ onset of annealing induced passivation changes, the shown lines are exponential fits of the data. Since FZ-Si material was used, the effective surface recombination velocity, $S_{\text {eff, }}$ may be approximated by $S_{\text {eff }}=d^{*}\left(2 \tau_{\text {eff }}\right)^{-1}$, with $d$ being the wafer thickness. The data are taken from [8]. single intrinsic a-Si:H film of about $50 \mathrm{~nm}$ thin (present on both wafer surfaces), for different values of $T_{\text {depo, }}$ changes as a function of the annealing temperature, $T_{\text {ann }}$. The substrates here are $3.0 \Omega . \mathrm{cm}$ p-type $\mathrm{FZ}$ wafers. For the films deposited at the lowest temperature $\left(T_{\text {depo }}=105^{\circ} \mathrm{C}\right)$, initially the passivation is poor, but improves to a remarkable extent by annealing. By increasing the deposition temperature, $T_{\text {depo, }}$ up to $180^{\circ} \mathrm{C}$, the surface passivation quality of as-deposited films improves. Here, post annealing up to $260^{\circ} \mathrm{C}$ increases the values for $\tau_{\text {eff }}$ further, well in excess of $1 \mathrm{~ms}$. This situation is different for films deposited at $205^{\circ} \mathrm{C}$. Annealing does not give rise anymore now to an improvement. For even higher values of $T_{\text {depo, }}$, the figure shows that the passivation quality actually goes down dramatically. Whereas the data in figure 1 was for still relatively thick $(50 \mathrm{~nm})$ intrinsic a-Si:H films with variable values for $T_{\text {depo, }}$ in figure 2 it is shown how the electronic passivation quality of much thinner (few $\mathrm{nm}) i-, p^{+}$- and $i / p^{+}$- structures changes by low temperature $\left(\leq 260{ }^{\circ} \mathrm{C}\right)$ postdeposition annealing. Here, $T_{\text {depo }}=155^{\circ} \mathrm{C}$. The table in the inset gives the values of the layer thickness $d_{\text {bulk }}$ and surface roughness $d_{\text {rough }}$ for the respective layers, as extracted again from SE measurements. For the $i$-case, the applied low temperature annealing treatment is again seen to have a beneficial influence on the passivation. This is different however for the $p^{+}$-case: here, annealing rapidly leads to passivation degradation. The latter situation is seen to be only slightly improved when an intrinsic buffer layer (of similar thickness as in the $i$-case) has been inserted underneath the $p^{+}$-film (i/p $p^{+}$-case): Starting with values similar as for the $p^{+}$-case, initially the passivation quality

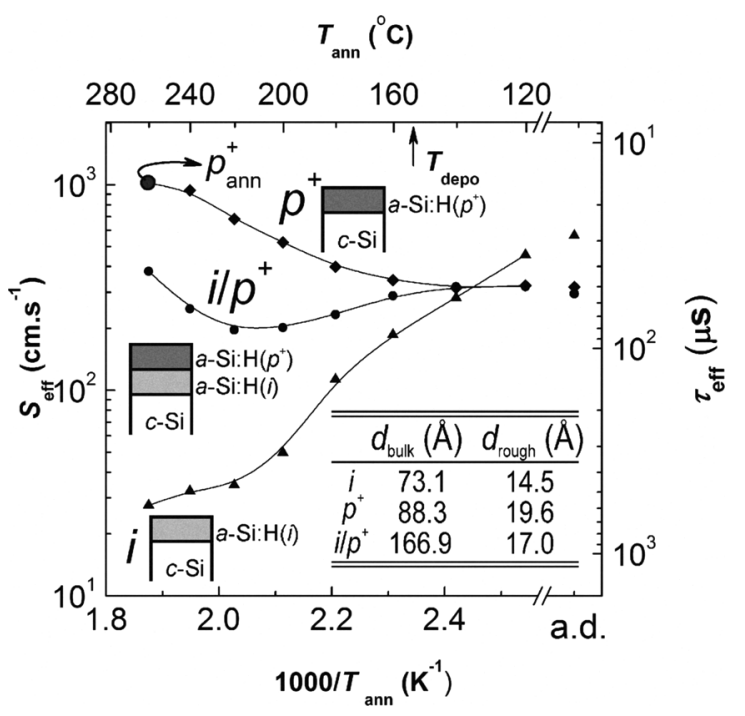

FIG. 2 Influence of $T_{\text {ann }}$ on the electronic passivation quality for thin intrinsic and doped a-Si:H layers deposited on mirror polished (100) FZ-Si $(n)$ surfaces. For reference, values for asdeposited films are given as well (label a.d. in the abscissa). The lines here are guides for the eye. The table in the inset gives the values of $d_{\text {bulk }}$, and $d_{\text {rough }}$ for the respective films, determined from SE measurements. The data are taken from [16] 
benefits from annealing. Nevertheless, from about $T_{\text {ann }}=$ $220^{\circ} \mathrm{C}$ on also here degradation sets in. Such trends have in the past been found to be irrespective of the dopanttype of low resistivity wafers.[6] We will now argue that the degradation mechanisms as seen in these two figures are of fundamentally different nature.

\section{DISCUSSION}

First we discuss the case of passivation by intrinsic a-Si:H films. From the slopes of the exponential fits in the shown Arrhenius plot in Fig. 1 , an activation energy, $E_{A}$, can be extracted. These values are given in figure 3 (b) as function of $T_{\text {depo. }}$. The area showing negative values for $E_{\mathrm{A}}$ corresponds to films for which post annealing is harmful for the passivation quality. This has been cross-hatched in the figure. Part (a) of the same figure gives the a-Si:H(i) film thickness, $d_{\text {bulk, }}$ during the initial deposition stages, as function of $T_{\text {depo. }}$. This data is obtained from fitting the measured SE data of the different samples to a two-layer model, taking the $50 \%$ void surface roughness on top of the a-Si:H film into account. For all films deposited at $T_{\text {depo }}$ $\geq 205^{\circ} \mathrm{C}$, hardly any film growth can be observed with SE during at least the first $72 \mathrm{sec}$. The difficulty to fit the initial film thickness to the SE data suggests that for the complete surface the deposited material is crystalline.[7] This area is cross-hatched in the figure too: A good

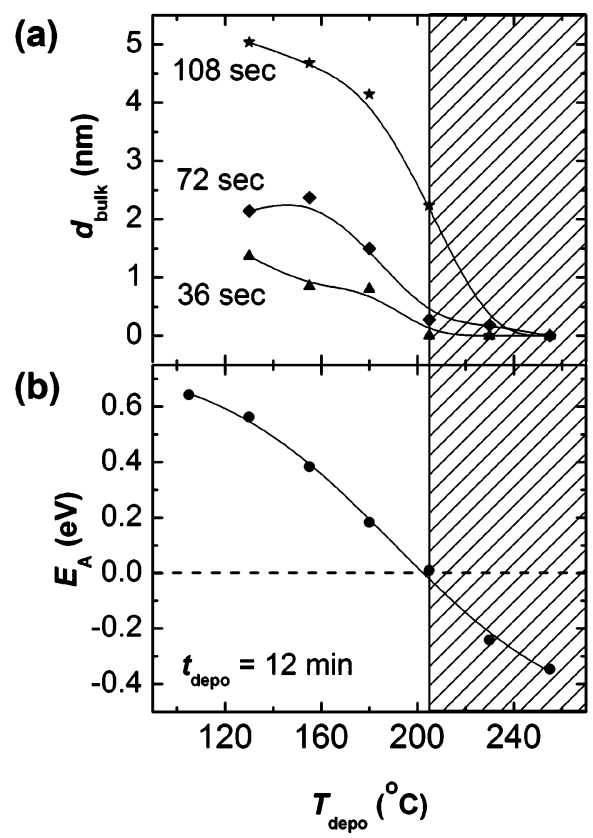

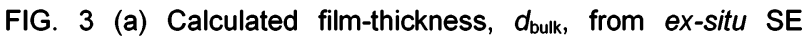
measurements as function of $T_{\text {depo }}$, given for several deposition times, $t_{\text {depo. }}$. Cross-hatched area shows films with epitaxially grown interface. (b) Extracted activation energy $E_{\mathrm{A}}$ as function of $T_{\text {depo }}$ for the films shown in figure 2. Cross-hatched area shows films for which the surface passivation degrades by annealing. All films are intrinsic. The lines are guides for the eye. The data are taken from [8]. correspondence exists between the onset where crystalline material is grown at the full interface during film deposition, and the point where the value of $E_{\mathrm{A}}$ becomes negative.[8]

The value for $E_{\mathrm{A}}$ is extracted from $\tau_{\text {eff }}$ measurements, hence its value will mainly depend on the density of electronically active defects of the film. These are the defects that are within reach of the $c$-Si minority carrier wave function. The impact of these defects is determined by their energetic position within the bandgap and their electron and hole capture cross sections. This contrasts with, e.g., electron spin resonance (ESR) measurements of a-Si:H(i) layers, which rather reveal paramagnetic defect densities in the bulk of relatively thick (typically a few $\mu \mathrm{m})$ films deposited on quartz substrates. Nevertheless, for $4 \mu \mathrm{m}$ thick films deposited at $25^{\circ} \mathrm{C}$, Biegelsen et al. observed in their ESR studies activation energies of about $0.5 \mathrm{eV}$ for the decrease of the $\mathrm{Si}$ dangling bond density by post deposition annealing at values for $T_{\text {ann }}$ up to $250^{\circ} \mathrm{C}$.[9] Considering the differences in measurement techniques, this value for $E_{\mathrm{A}}$ corresponds well with the ones obtained from $\tau_{\text {eff }}$ measurements for the films deposited at the lowest values $T_{\text {depo }}$ as presented in figure 3 (a).

For higher values of $T_{\text {depo, }}$ the value of $E_{A}$ lowers and eventually passes through zero. Likely, this is because by increasing $T_{\text {depo }}$ the deposited material contains a lower dangling bond density. When $E_{\mathrm{A}}$ equals to zero there is no decrease in dangling bond density any more. For negative

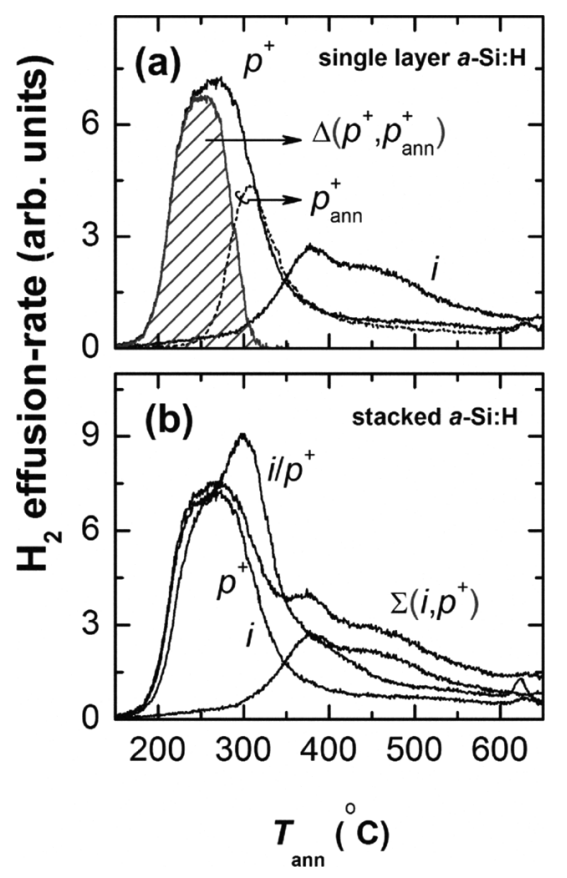

FIG. 4 Influence of $T_{\text {ann }}$ on $\mathrm{H}_{2}$ effusion rate of a-Si:H films as given in Fig. 1: (a) data for few $\mathrm{nm}$ thin single layer a-Si:H films, as deposited and after the low-temperature annealing cycle described in Fig. 1 and 2. (b) data for as-deposited stacked 
doped films. Measurements by TDS. The data are taken from [16].

values of $E_{\mathrm{A}}$ annealing increases the dangling bond density.

These results suggests that at all values for $T_{\text {depo }}$ two competing mechanisms may be at work during annealing, resulting in an in- and decrease of the dangling bond density, respectively. These two mechanisms likely depend on how much hydrogen is available in the film for dangling bond passivation. This may explain why negative values of $E_{A}$ correspond to epitaxially grown films, where it can be expected that the amount of available hydrogen is limited, and where it is known that such growth during a$\mathrm{Si}: \mathrm{H}(i)$ deposition is detrimental for heterojunction device performance. $^{10}$ This result also shows that annealing combined with carrier lifetime measurements offers an alternative means, compared to transmission electron microscopy (TEM) or SE,[11] to determine the abruptness of the interface.[8]

To explain the experienced degradation in case of boron-doped films, first we show in figures 4 (a) and (b) TDS data of exactly the same structures as displayed in Fig. 2. Part (a) shows that for such thin films, the boron doping ( $p^{+}$-case) leads to $\mathrm{H}_{2}$ effusion at significant lower temperatures shown compared to intrinsic films, occurs. This phenomenon has been reported earlier in literature for thicker films.[12] The same figure also shows similar data (label $p_{a n n}^{+}$) for the doped film case after the stepwise annealing cycle, as described in Fig. 2 . The crosshatched area in this figure (label $\Delta\left(p^{+}, p_{a n n}^{+}\right)$) represents the difference between these two signals and clearly demonstrates that during the latter cycle already significant $\mathrm{H}_{2}$ effusion takes place. Figure 4 (b) compares $\mathrm{H}_{2}$ effusion rate data of the ilp $p^{+}$-structure with that of the summed $i$ - and $p^{+}$-case (label $\Sigma\left(i, p^{+}\right)$). It is seen that at low temperatures more hydrogen effuses out for the ilp ${ }^{+}$case than for the combined $i$ - and $p^{+}$-cases. Note that the combined $i$ - and $p^{+}$-layer thickness practically equals that of the stacked ilp ${ }^{+}$structure (see inset in Fig. 2).

For intrinsic a-Si:H material annealed at higher temperatures $\left(>300^{\circ} \mathrm{C}\right)$, a correspondence between the $\mathrm{H}_{2}$ effusion rate and defect-generation in the film has been demonstrated in the past by comparing TDS, IR absorption and electron spin resonance measurements.[9] Consequently, for boron-doped a-Si: $\mathrm{H}_{\left(p^{+}\right)}$material, the effusion data suggests that hydrogen likely is already transferred at much lower temperatures from a $\mathrm{Si}-\mathrm{H}$ to a $\mathrm{H}_{2}$ state, creating defects in the material. The $\mathrm{Si}-\mathrm{H}$ bond rupture energy has been argued to depend on the Fermi energy (rather than on the actual dopants) in such material.[13,14] As discussed, in the present case, annealing rapidly results in electronic passivation losses for the $p^{+}$-case. Also here, the origin of this phenomenon likely is Fermi-level dependent defect-generation, occurring close to the a-Si:H / c-Si interface.

For the i/p $p^{+}$-structure, the initial improvement by annealing in passivation is again most probably due to outannealing of defects in the intrinsic layer. At higher values for $T_{\text {ann }}$, the passivation degradation of this stacked structure may be caused by two related phenomena: Firstly, dangling bonds are created already at low temperatures by annealing in the doped layer. As a consequence, the wavefunction associated with the minority carriers close to the interface in the $c$-Si material may probe through the intrinsic film, when sufficiently thin, to collapse in the defect rich doped overlayer.[15] Secondly, in addition to this, due to the presence of the $p^{+}$layer on top of the $i$-layer, also in the latter layer the Fermilevel will be shifted towards the valence band of the material. As a result, also here already at moderate annealing temperatures, $\mathrm{Si}-\mathrm{H}$ bond-rupture can be expected to take place in the buffer layer too.[16] This is evidenced in Fig. 4 (b), where it can be seen that at lower temperatures more $\mathrm{H}_{2}$ effuses out of the stack than for the two films measured separately. Conversely, at higher temperatures a smaller amount effuses out. Consequently, it must be concluded that the presence of a $p^{+}$-type overlayer likely enhances $\mathrm{Si}-\mathrm{H}$ bond rupture in the intrinsic buffer layer. As a result, also for the $c-\mathrm{Si} / \mathrm{a}-\mathrm{Si}: \mathrm{H}(i) /$ a-Si: $H\left(p^{+}\right)$structure, high dangling bond densities may be generated not only in the doped over layer, but also close to the $c$-Si / a-Si:H interface, already at relatively low values for $T_{\text {ann }}$.

\section{CONCLUSIONS}

In this article, we discussed how interface properties of a$\mathrm{Si}: \mathrm{H} / \mathrm{c}$-Si structures may determine the electronic passivation behaviour. Post annealing treatments have been found to be a good tool to unravel the physical mechanism of such passivation. In addition, the described phenomena demonstrate the need for a careful assessment of temperature treatments during $c-\mathrm{Si} / \mathrm{a}-\mathrm{Si}: \mathrm{H}$ heterostructure device fabrication. For intrinsic film deposition, the deposition temperature should be sufficiently low to prevent epi-Si growth. For doped layer deposition, care has to be taken not to generate harmful defects already at moderate temperatures.

[1] J.I. Pankove and M.L. Tarng, Appl. Phys. Lett. 34, 156 (1979).

[2] H. Matsuura, et al., J. Appl. Phys. 55, 1012 (1984).

[3] M. Tanaka, et al., Jpn. J. Appl. Phys. 31, 3518 (1992).

[4] Y. Tsunomura, et al., Technical digest of the 17th International Photovoltaic Science and Engineering Conference, Fukuoka, Japan, 2007, p. 387.

[5] R.A. Sinton and A. Cuevas, Appl. Phys. Lett. 69, 2510 (1996).

[6] S. De Wolf and M. Kondo, in Proceedings of the $4^{\text {th }}$ World Conference on Photovoltaic Energy Conversion, Waikoloa, Hawaii (IEEE, Piscataway, NJ, 2006), p. 1469.

[7] H. Fujiwara and M. Kondo, Appl. Phys. Lett. 90, 013503 (2007).

[8] S. De Wolf and M. Kondo, Appl. Phys. Lett. 90, 042111 (2007).

[9] D.K. Biegelsen, et al., Phys. Rev. B 20, 4839 (1979).

[10] T.H. Wang, et al., Thin Solid Films 501, 284 (2006).

[11] D.H. Levi, et al., J. Vac. Sci. Technol. A 24, 1676 (2006).

[12] W. Beyer, et al., Solid State Comm. 39, 375 (1981).

[13] R.A. Street, et al., Philos. Mag. B 56, 305 (1987).

[14] W. Beyer, Physica B 170, 105 (1991).

[15] S. De Wolf and G. Beaucarne, Appl. Phys. Lett. 88, 022104 (2006).

[16] S. De Wolf and M. Kondo, Appl. Phys. Lett. 91, 112109 (2007). 